\section{Transformation-invariant cues in the}

\section{recognition of simple visual patterns*}

\author{
JOSEPH S. LAPPIN \\ Vanderbilt University, Nashville, Tennessee 37203
}

Two alternative hypotheses about the discriminative cues in visual patterns were tested by comparing the speed with which Ss could apply two different classification rules for identifying a set of 16 simple dot patterns, consisting of four groups of four transformations. One rule required discrimination between groups of transformations, and another rule required discrimination between transformations within groups. The patterns within a transformation group were less similar in their positioning of dots than were patterns of the same transformation in different groups. Speed of identification, however, was more rapid for the discrimination between groups than for the discrimination of transformations within groups and was also invariant with respect to the specific transformations included in the same category under the between-groups classification rule. The discriminative cues in these patterns were thus indicated to be relationships among dots that remained invariant under the group of transformations.

The recognition of visual patterns may be considered as a classification process : a large set of two-dimensional retinal stimulus patterns is partitioned into smaller subsets such that different environmental stimuli are classified into different subsets. A central problem in the study of this process concerns the achievement of "perceptual constancy," whereby a large subset of "proximal" (retinal) stimulus patterns that originate from the same "distal" (environmental) stimulus object produces a constant perception of the same object. A given distal stimulus object produces a widely varying set of proximal patterns on the retina, depending upon the distance, viewing angle, and relative motion of the observer, and upon such environmental conditions as illumination and the presence of other objects. As these conditions are changed, a given pattern may undergo a variety of planar and projective transformations and may be embedded in various irrelevant contexts.

Two general classes of models for pattern recognition differ in their assumptions about the discriminative cues that are detected in the proximal stimulus patterns. The prevalent assumption is that independently variable features of the distal object provide the input cues for discriminating one object from a nother. Although the individual component cues are unreliable indicators of their distal origin, the constraints on the complete set of redundant cues in a proximal pattern should be expected to provide a reliable index of the distal object. This assumption was suggested some time

*The author is grateful to Thomas J. Hicks for his assistance in collecting this data. ago in Helmholtz's theory of "unconscious inferences" and later in Brunswik's (1952) "lens model" of perceptual achievements. A more detailed account of the role of redundancy in the perception of structured patterns has been provided by Garner's (1962) careful application of information theory to these problems.

An alternative but infrequent assumption is that the discriminative cues in the proximal pattern are based on complex geometric properties that remain invariant under the group of planar and projective transformations of patterns evoked by a given distal object. As James Gibson $(1950,1966)$ has pointed out, such cues are provided by the spatial and temporal relations among the component elements of two-dimensional patterns arising from objects moving in three-dimensional space. By this assumption, the task of explaining perceptual constancy becomes one of specifying the geometric measures that remain invariant under a group of transformations and of identifying the processes for computing these invariant measures. Pick (1965) has provided evidence that transformation-invariant cues are acquired in learning to identify unfamiliar shapes.

The question of which cues provide the discriminative basis for recognizing visual patterns was studied in the present experiment by measuring the speed with which Ss could identify simple patterns according to several specified rules of classification. The principal assumption is that the speed of identification reflects the amount of perceptual processing required to detect the properties that discriminate among the subsets of patterns defined by the classification rule. A second assumption is that if the identification speed is independent of variation in the patterns included within a particular classified subset, then the discriminative cue is invariant over that subset of patterns. Thus, the speed for classifying the patterns into various subsets was used to infer the perceptually effective cues for discriminating among the patterns. The set of patterns used in this experiment was composed of groups of dot patterns such that each pattern within a group could be transfor. ed into any other pattern in the sume group by a rotation or reflection. Ss were asked to identify patterns from different transformation groups or to identify patterns within the same transformation group; additionally, the classified subsets contained either a single pattern or contained several different patterns. One obtains distinctly different predictions about the relative speed for classifying this particular set of patterns, depending on whether one assumes the discriminative cues to consist of the "lower order" physical variables or of "higher order" relationships among the component physical variables.

This experiment was motivated by a previous (unpublished) experiment using the same set of patterns in which it was unexpectedly found that the speed for identifying patterns in different transformation groups was approximately independent of how many and which transformations were included within a single classified subset. This result suggested that the discriminative cues for recognizing the patterns were invariant over the group of rotations and reflections. The purpose of the present experiment was to verify this result by also determining the speed of identifying patterns within the same transformation group.

\section{Stimuli}

\section{METHOD}

The stimulus set, shown in Fig. 1, consisted of 16 dot patterns formed in a 3 by 2 matrix of possible dot positions. In each position a black dot was either large (1/16-in. diam) or small (1/32-in. diam); the distance between vertically adjacent dots was $1 / 6$ in. o.c.; the distance between horizontally adjacent dots was $1 / 4$ in. Each pattern was centered on a $3 \frac{1}{2} \times 2 \frac{3}{4}$ in. card. (The cards were originally constructed for training teachers of the blind and contained scaled representations of braille patterns.)

The set of 16 patterns was composed of four groups (in the mathematical sense) of four patterns such that each pattern within a group could be transformed into any other 


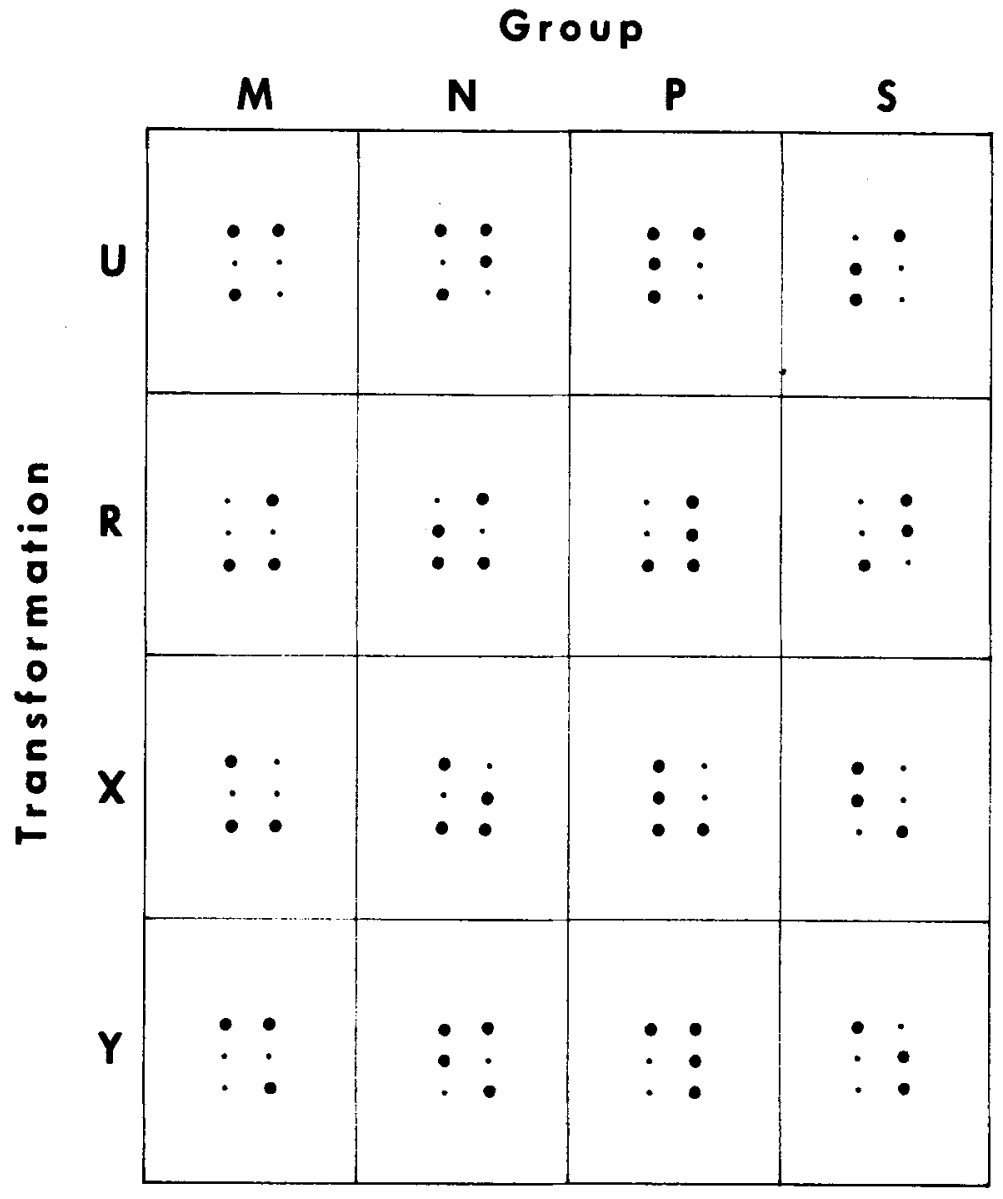

Fig. 1. Stimulus patterns.

pattern of that group by a rotation of $180 \mathrm{deg}$ or a reflection about either the horizontal or vertical axis. For convenience in labeling the patterns, one pattern in each group was referred to as " $U$ " (for upright), another as " $R$ " (rotation of $U$ ), another as " $X$ " (reflection of $U$ about the $X$ axis), and another as " $Y$ " (reflection of $U$ about the $Y$ axis). The four groups of patterns were designated as " $M$," " $N$," "P," and "S." (The U patterns of the four groups corresponded to the braille code for $M, N, P$, and $S$.)

The time required to identify these patterns is presumably a decreasing function of the perceptual discriminability of the subsets to be classified and of the similarity of patterns within these subsets. Measurements of the differences among these subsets are therefore essential to predictions of the Ss' performance. The six dot positions in the 3 by 2 matrix offer an obvious basis for these measurements each position may be treated as a binary variable that contains either a large or a small dot. A convenient measure of the overall discriminability among a set of patterns is provided by the information content $\left(-\Sigma\left[p_{i} \log _{2} p_{i}\right]\right)$ less obvious for conditions in which several nonidentical patterns are included within a single classified subset.

The preceding measures of discriminability assume that the perceptual cues for recognizing patterns are based on the six variable positions in which dots occur. Many alternative assumptions are possible. One alternative is that the discriminative properties are Gestalt-like features that remain invariant over the group of transformations. Such features can be defined on pairs of dots: The horizontal and vertical distances between any two dots remain constant under the group of rotations and reflections used in this study. If this set of interdot distances was a primary discriminative basis for identifying these patterns, then the identification of patterns in different transformation groups should be faster than the within-groups identification and should also be unaffected by variation of patterns within transformation groups.

\section{Classification Rules}

Ss identified patterns according to two different classification rules under 10 conditions. Five conditions required Ss to identify patterns between groups and five conditions required identification of patterns within groups. In four of the between-groups conditions the transformation was held constant- $U$, $\mathrm{R}, \mathrm{X}$, or $\mathrm{Y}-$ and in a fifth condition all 16 patterns occurred equally often. In a corresponding set of five within-groups conditions, the groups were held constant-M, $N, P$, or $S$-or all 16 patterns again occurred with equal frequency. Thus, in the between-groups conditions Ss identified the patterns as "M," "N," "P," or " $S$," and in the within-groups conditions the same 16 patterns were identified as " $U$," " $R$," "X," or "Y."

\section{Procedure}

Three paid and practiced female volunteers served as Ss. Each S served for 10 sessions. The between-groups and within-groups classification rules were used in alternate sessions; for two of the Ss the between-groups classification rules were used in the odd-numbered sessions, and for the other $S$ the between-groups classification rules were used in the even-numbered sessions. At the beginning of the experiment, $S$ was presented with the "upright" transformation from each of the four groups and was asked to learn the labels for these patterns. She was also shown the other transformations of the patterns and was asked to learn the 
labels for these transformations.

On each trial $\mathbf{S}$ was given a shuffled pack of 32 cards and asked to identify the patterns as rapidly as possible, according to the classification rule for that session. A stopwatch was used to measure the time required to identify all 32 patterns in each card pack. The Ss were asked not to make errors, and none was detected by the $\mathrm{E}$. At the three practice trials to familiarize herself with the classification rule for that session. Each of the 16 patterns appeared twice within each of these practice trials. The next 10 trials were experimental trials; in the first five trials, each of the five variations on the classification rule (four conditions with one pattern per category and a fifth condition with all four patterns in each category) occurred once, and in the next five trials these conditions occurred in the reversed order. The order of occurrence of conditions was counterbalanced for each $S$ and was varied across Ss. An entire session required about $30 \mathrm{~min}$.

The data that were analyzed and presented below were taken only from the last six sessions, yielding a total of six trials per $S$ per condition. The decision to utilize only the last six sessions was based on the occurrence of large practice effects in the earlier sessions. Performance was more nearly stable after the first four sessions, although some continued improvement was evident in the later sessions. The large differences between conditions were, however, even larger in the early than they were in the late sessions.

\section{RESULTS}

Table 1 gives the average time taken by each $S$ to identify the set of 32 patterns under each classification rule and condition. As is evident, the times for between-groups identifications were much faster than were those for the within-groups identifications. Additionally, differences among the five between-groups conditions were small and inconsistent, whereas reliable differences were obtained among the within-groups conditions. beginning of each session, $S$ was given

Analyses of variance for the effects of between-groups vs within-groups classification rules yielded $\mathrm{F}$ ratios of 57.35, 25.49, and 14.35 for Ss R.D., A.C., and A.Y., respectively [for $p \leqslant .01, F(1 / 10)=10.04] . F$ ratios for the differences among between-groups conditions were $1.13,2.26$, and 2.81 for R.D., A.C., and A.Y. [for $p \leqslant .05$, $F(4 / 20)=2.87]$. F ratios for the within-groups conditions were 16.32 , 24.54 , and 8.54 [for $p \leqslant .01$, $F(4 / 20)=4.43]$.

The magnitude of these effects was surprisingly large and consistent, both for individual Ss and across Ss. The only exception to this consistency occurred in the withingroups conditions: For each $\mathbf{S}$, patterns were identified at significantly different speeds in these five conditions, but the relative speeds for the conditions varied from one $S$ to another. Differences in the speeds for the within-groups conditions had been anticipated, on the hypothesis that no single cue would permit discrimination among the patterns in every condition and that some discriminative cues should require more processing time than others. By this reasoning, longer times had been expected for the condition in which all four groups were included in the same classified category. The longest average identification times were indeed obtained in this condition, but this was not true for one S. S R.D. consistently gave the longest times in identifying the transformations in Group S; for A.Y., also, this condition produced longer times than other conditions involving a single group of patterns. In all of these within-groups conditions there are several alternative cues by which patterns could potentially be recognized; different Ss probably selected different cues. There should be more alternative choices for selection in Condition $S$ than in the other single-group conditions, since the information content of the six dot positions is greatest in this condition. Rather than improve the discriminability of patterns, redundancy may under some conditions contribute to confusion in

Table 1

Average Times (Seconds) for Identifying 32 Patterns for Each $S$ and Each Condition Classification Rule

\begin{tabular}{|c|c|c|c|c|c|c|c|c|c|c|}
\hline \multirow[b]{4}{*}{ Ss } & \multicolumn{10}{|c|}{ Classification Rule } \\
\hline & \multicolumn{5}{|c|}{ Between Groups } & \multicolumn{5}{|c|}{ Within Groups } \\
\hline & \multicolumn{5}{|c|}{ Transformation } & \multicolumn{5}{|c|}{ Group } \\
\hline & $\mathbf{U}$ & $\mathbf{R}$ & $\mathbf{X}$ & $\mathbf{Y}$ & All & $\mathbf{M}$ & $\mathbf{N}$ & $\mathbf{P}$ & $\mathbf{S}$ & All \\
\hline RD & 22.9 & 22.9 & 24.9 & 24.8 & 23.5 & 33.1 & 35.3 & 32.7 & 50.4 & 42.6 \\
\hline $\mathrm{AC}$ & 19.5 & 20.3 & 20.2 & 20.2 & 19.0 & 26.2 & 28.6 & 26.1 & 25.5 & 38.6 \\
\hline AY & 21.6 & 23.1 & 23.0 & 23.8 & 21.3 & 26.9 & 28.6 & 27.8 & 31.4 & 34,6 \\
\hline Average & 21.4 & 22.1 & 22.7 & 22.9 & 21.3 & 28.7 & 30.8 & 28.9 & 35.8 & 38.6 \\
\hline
\end{tabular}

attending to a particular cue.

\section{DISCUSSION}

The principal finding of this experiment is that some patterns are recognized on the basis of features that are invariant over a group of planar transformations. For many readers, this finding may have little "surprise value," since the patterns in the same group look more alike than the patterns in different groups and since no one has ever explicitly questioned the hypothesis that transformation-invariant properties are important in form perception. The contribution of this experiment derives from its contradiction of the commonly accepted theory that form perception is a process for utilizing the redundancy in a set of variables (e.g., Gamer, 1962, 1966). The component variables of these patterns (i.e., the dots) are more redundant in the classification of transformations within the same group than in the classification of the same transformation from different groups. On the hypothesis that psychological dissimilarity and discriminability increase with redundancy, the speed of recognition should be greater for the classification of transformations within a group. But this hypothesis clearly failed as an explanation for the speed of recognition for these patterns. The discriminative cues for this set of patterns must have been provided by relationships among the component dots.

The distinction between component elements and the relationships between them sounds like a play on words, but is in fact more fundamental. Relationships may be defined independently of the specific physical components -e.g., larger, next to, above, darker, etc. - and may be recursively applied to generate a simple inductive description of an infinite series of components e.g., $a$ spot of light is at position $p$ at time $t$ and moves $1 \mathrm{~mm}$ to the right at each successive millisecond. The distinction between component elements and the recursive relations between them is analogous to the distinction in linguistic theory between a list structure and a generative grammar (see Chomsky, 1963). Restle (1970) has also recently emphasized the importance of the distinction between associative and recursive theories of serial pattern learning. The limited capabilities of pattern recognition systems utilizing a linear combination of component cues have been elegantly analyzed by Minsky and Papert (1969).

A fundamental aspect of any theory of perception or information processing concerns the representation 
of input stimulus data. Theories of information processing are tied to assumptions about the units of information being processed. Research and theory on human perception have, however, seldom sought to explicate or justify assumptions about the representation of stimulus input. The work of James Gibson (e.g., 1960, 1966) is a notable exception. The prevailing practice has been to operationally represent the stimulus input by those physical variables from which the stimuli were constructed. But, as the present experiment demonstrates, this assumption may often mislead. A similar demonstration has been provided by Lappin, Snyder, and Blackburn (1971). The point is that we have as yet little a priori basis for assuming that a specific variable provides the discriminative cue for human perception.

A large number of relationships would suffice to account for the speed of recognition of the specific patterns and transformations used in this study. The simplest class of sufficient relations may be defined on pairs of dots. One example of such a relational representation of these patterns may be constructed from the "spectrum" of horizontal and vertical distances between pairs of dots; there are five such relations, consisting of dots separated by one unit of vertical distance, by two units of vertical distance, by one unit of horizontal distance, by one horizontal and one vertical unit, or by one horizontal and two vertical units of distance. This set of relations actually provides more cues than necessary for discriminating among the specific patterns in this study; it would suffice to consider only the vertical distances between dots in the same column with no horizontal separation. Since a number of alternative representations of the discriminative cues in these patterns are sufficient but not necessary to account for the Ss' performance, the specific perceptually effective cues cannot be identified. We may conclude only that the discriminative cues are defined on two or more dots.

The important question raised by these results concerns the generalization to other patterns and other transformations. One wonders, for example, about the discriminative cues available in patterns which contain lines and angles, which contain multiple shapes and brightnesses, or which contain perspective and stereoscopic depth. Similarly, it is interesting to consider transformations in time, projections from rigid transformations in three-dimensional space, or distortion produced by stochastic processes. As the set of patterns and the group of transformations is varied, discriminative cues remaining invariant over the transformation group are changed in complexity-i.e., changed in the minimal number of elements or area on which the cues are defined, or in the amount of computation necessary to detect the cues.

If the recognition of these simple visual patterns was based upon features that remain invariant over reflections and rotations, then the data processing in the peripheral visual system must initially extract relational properties or Gestalts. The extraction of details in specific positions seems to require more prolonged processing of the pattern. This conclusion differs from Kolers and his associates' (1968 $1969 \mathrm{a}, 1969 \mathrm{~b})$ conclusion that visual pattern recognition depends upon an orientation-detection process. Kolers' experiments demonstrated that the speed for reading transformed print depends upon the orientation of the printed words and of the letters within words. The apparent discrepancy with Kolers' studies indicates an examination of differences in method. First, Kolers' manipulations correspond to the set of between-groups conditions in this experiment; his Ss were not asked to identify the orientation of the print, only to read words or letters. If the identification of orientation were more difficult than the identification of shape, as in the present experiment, then the importance of orientation-sensitive processes would seem lessened. Second, some printed letters (e.g., b, d, p, q) become identical or similar by the application of a rotation or reflection. Insofar as this condition holds, then the dependence of reading time on orientation may result not from sensitivity to orientation but from confusability of different orientations. Third, Kolers employed a much larger stimulus set containing stimuli of quite different physical characteristics from those in this study. His stimulus materials offer advantages in generalizing to other patterns, but concomitant disadrantages in controlling the similarity between stimuli in the same or in different orientations. Fourth, Kolers' findings indicated that the recognition of linear arrays of textual material utilizes additional context-sensitive processes beyond the recognition of single characters. At any rate, the discrepancy between this experiment and those of Kolers demands further study of the conditions under which transformations alter perceptual processing.

The results of this study reflect on certain phenomena in children's reading. Children with reading problems are commonly observed to confuse letters and words which differ by a reflection about the vertical axis (see Howard \& Templeton, 1966). A common conclusion is that the reading problems have resulted from a disability in orientation detection processes (e.g., Orton, 1937). An alternative conclusion is that confusions in orientation are the result of the reading problem. The relative difficulty of identification of transformations within groups, experienced by the adult $S$ s in this experiment, indicates that identifying orientation is more difficult than other perceptual processes and is therefore more likely to be affected by reading problems. Interestingly, laboratory rats also have difficulty in discriminating figures which differ by a reflection about the vertical axis (Kinsbourne, 1971).

\section{REFERENCES}

BRUNSWIK, E. The conceptual framework of psychology. In International encyclopedia of unified science. Vol. 1. Chicago: University of Chicago Press, 1952.

CHOMSKY, N. Formal properties of grammars. In R. D. Luce, R. R. Bush, and E. Galanter (Eds.), Handbook of mathematical psychology. Vol. II. New Yonk: Wiley, 1963.

GARNER, W. R. Uncertainty and structure as psychological concepts. New York: Wiley, 1962.

GARNER, W. R. To perceive is to know. American Psychologist, 1966, 21, 11-19. GIBSON, J. J. Perception of the visual world. Boston: Houghton Miffin, 1950.

GIBSON, J. J. The concept of the stimulus in psychology. American Psychologist, $1960,15,694-703$.

GIBSON, J. J. The senses considered as perceptual systems. Boston: Houghton Mifflin, 1966.

HOWARD, I. P., \& TEMPLETON, W. B. Human spatial orientation. New York: Wiley, 1966.

KIN S BO URNE, M. Discrimination of orientation by rats. Psychonomic Science, $1971,22,50-51$.

KOLERS, $P$. A. The recognition of geometrically transformed text. Perception \& Psychophysics, 1968, 3, 57-64.

KOLERS, P. A., \& PERKINS, D. N. Orientation of letters and errors in their recognition. Perception \& Psychophysics, $1969 a, 5,265-269$

KOLERS, P. A., \& PERKINS, D. N. Orientation of letters and their speed of recognition. Perception \& Psychophysics, 1969 b. 5, 275-280.

LAPPIN, J. S., SNYDER, C. R., \& BLACKBURN, C. The encoding of perceptual information in the organization of individual stimulus patterns. Perception \& Psychophysics. $1971,10,123-128$.

MINSKY, M., \& PAPERT, S. Perceptrons. Cambridge: M.I.T. Press, 1969.

ORTON, S. T. Reading, writing, and speech problems in children. New York: Norton, 1937.

PICK, A. D. Improvement of visual and tactual form discrimination. Journal of Experimental Psychology, 1965, 69. 331-339.

RESTLE, F. Theory of serial pattern learning: Structural trees. Psychological Review, 1970, 77, 481-495.

(Accepted for publication May 17, 1971.) 